\title{
Influence of the nonlinearity on statistical characteristics of long
}

\section{wave runup}

\author{
P. Denissenko ${ }^{1}$, I. Didenkulova ${ }^{2,3}$, E. Pelinovsky ${ }^{4}$, and J. Pearson ${ }^{1}$ \\ ${ }^{1}$ School of Engineering, University of Warwick, Coventry, CV4 7AL, UK \\ ${ }^{2}$ Laboratory of Wave Engineering, Institute of Cybernetics, Akadeemia tee 21, 12618 Tallinn, Estonia \\ ${ }^{3}$ Nizhny Novgorod State Technical University, Minin str. 24, 603950, Nizhny Novgorod, Russia \\ ${ }^{4}$ Institute of Applied Physics, Uljanov str. 46, 603950 Nizhny Novgorod, Russia
}

Received: 10 October 2011 - Revised: 1 December 2011 - Accepted: 5 December 2011 - Published: 14 December 2011

\begin{abstract}
Runup of long irregular waves on a plane beach is studied experimentally in the water flume at the University of Warwick. Statistics of wave runup (displacement and velocity of the moving shoreline and their extreme values) is analyzed for the incident wave field with the narrow band spectrum for different amplitudes of incident waves (different values of the breaking parameter $\mathrm{Br}_{\sigma}$ ). It is shown experimentally that the distribution of the shoreline velocity does not depend on $\mathrm{Br}_{\sigma}$ and coincides with the distribution of the vertical velocity in the incident wave field as it is predicted in the statistical theory of nonlinear long wave runup. Statistics of runup amplitudes shows the same behavior as that of the incident wave amplitudes. However, the distribution of the wave runup on a beach differs from the statistics of the incident wave elevation. The mean sea level at the coast rises with an increase in $\mathrm{Br}_{\sigma}$, causing wave set-up on a beach, which agrees with the theoretical predictions. At the same time values of skewness and kurtosis for wave runup are similar to those for the incident wave field and they might be used for the forecast of sea floods at the coast.
\end{abstract}

\section{Introduction}

The prediction of possible flooding and properties of the water flow on the coast is an important practical task for physical oceanography and coastal engineering, which results in numerous empirical formulas describing runup characteristics of wind waves and swell available in the engineering literature (see, for instance, Le Mehaute et al., 1968; Stockdon et al., 2006). Very often these formulae are strongly dependent on the site specific location of the coastal zone due to effects of reflection, refraction and diffraction. In the

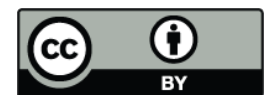

Correspondence to: P. Denissenko (p.denissenko@gmail.com) deterministic approach for a solitary incident wave the process of wave runup is modelled within fully-nonlinear Euler or Navier-Stokes equations including effects of wave breaking and dissipation in the near-bottom boundary layer (Liu et al., 1995; Kennedy et al., 2000; Choi et al., 2007, 2008; Fuhrman and Madsen, 2008). In the case of an irregular incident wave field the wave runup on a beach is usually calculated from empirical expressions (Massel, 1989), which can be found from experimental studies in laboratory and natural conditions. The statistics of nonlinear runup of irregular breaking waves was reported by Hedges and Mase (2004) who conducted laboratory investigations. Experimental studies of wave runup on a beach in natural conditions have been undertaken by numerous investigators (e.g. Bowen et al., 1968; Huntley et al., 1977; Guza and Thornton, 1980; Holman and Sallenger, 1985; Holman, 1986; Raubenneimer and Guza, 1996; Raubenneimer et al., 2001). It has been shown that nonlinearity in the coastal zone leads to an increase in the mean sea level at the coast (wave set-up) for any distribution of the wave field (Bowen et al., 1968; Huntley et al., 1977; Raubenneimer and Guza, 1996; Dean and Walton, 2009) and that the distribution of wave runup on a beach deviates from a Gaussian profile (Huntley et al., 1977).

Runup of irregular non-breaking waves was theoretically studied by Didenkulova et al. (2011), where the nonlinear shallow water theory was applied to beaches of constant slope. Beach slopes of constant inclines are commonly used in validation techniques, as it enables an exact solution of the nonlinear shallow water theory to be established (Carrier and Greenspan, 1958). In the statistical approach, Didenkulova et al. (2011) have found relationships between distributions of wave runup, shoreline velocity and statistics of the incoming irregular wave field. Didenkulova et al. (2011) demonstrated that the nonlinearity does not change the statistics of the shoreline velocity, but does influence the statistics of wave runup displacement, resulting in a change to its statistical moments. In this paper the influence of the nonlinearity

Published by Copernicus Publications on behalf of the European Geosciences Union and the American Geophysical Union. 
on the statistics of wave runup is studied experimentally and compared with the theoretical predictions.

The paper is organized as follows. The shallow water theory and the main theoretical results are briefly discussed in Sect. 2. The experimental setup is described in Sect. 3. The structure of the incident wave field is presented in Sect. 4. The experimental results on statistics of wave runup are discussed in Sect. 5, culminating with conclusions in Sect. 6.

\section{Scientific background}

The statistics of irregular wave runup on a plane beach is studied within the framework of nonlinear shallow water theory

$\frac{\partial \eta}{\partial t}+\frac{\partial}{\partial x}[(h+\eta) u]=0, \quad \frac{\partial u}{\partial t}+u \frac{\partial u}{\partial x}+g \frac{\partial \eta}{\partial x}=0$,

where $\eta(x, t)$ is water displacement, $u(x, t)$ is depthaveraged velocity, $h(x)$ is unperturbed water depth, $g$ is gravity acceleration, $x$ is a coordinate, directed onshore, and $t$ is time. The beach is assumed to be plane $h(x)=-\alpha x$, where $\alpha$ is a constant beach slope. The main conclusion of the nonlinear shallow water theory based on Eq. (1) is that extreme runup statistics (maximum runup and backwash heights and maximum runup and backwash velocities of the shoreline) in nonlinear and corresponding linear theories (extremes of sea level oscillations $R(t)$ and velocity $U(t)$ in the point $x=0$ for the linearized Eq. (1) coincide if an incident wave approaches the shore from far distance (Carrier and Greenspan, 1958; Synolakis, 1991; Didenkulova et al., 2008). For the case of irregular waves, Didenkulova et al. (2011) demonstrated that this theory is still valid.

For example, if initial wave field is represented by a Gaussian stationary random process with a narrow-band spectrum, the distributions of amplitudes of the nonlinear wave runup are described by Rayleigh distribution

$f\left(R_{\text {extr }}\right)=\frac{4 R_{\text {extr }}}{R_{\mathrm{s}}^{2}} \exp \left(-2\left[\frac{R_{\text {extr }}}{R_{\mathrm{s}}}\right]^{2}\right)$,

where $R_{\text {extr }}$ is the extreme (maximum or minimum) runup heights, $R_{\mathrm{S}}$ is the significant runup height, defined as the averaged of $1 / 3$ of the largest waves, which is often used in the oceanography; for the Gaussian process $R_{\mathrm{S}}=4 \sigma_{R}, \sigma_{R}$ is the standard deviation of linear wave runup.

The distribution Eq. (2) can also be written in terms of the shoreline velocity using the significant shoreline velocity amplitude $U_{\mathrm{s}}$. Moreover, the distribution functions and, hence, statistical moments of the nonlinear velocity of the moving shoreline $u(t)$ coincide with distribution functions and statistical moments of the corresponding velocity in the linear problem $U(t)$ :

$<u^{n}>=<U^{n}>$.
Therefore, the nonlinearity does not influence the statistics of the shoreline velocity. In contrast to the shoreline velocity, the distribution of wave runup is not Gaussian and is influenced by nonlinearity. Statistical moments of the nonlinear wave runup $r(t)$ can be derived through standard deviations of linear wave runup $\sigma_{R}$ and displacement velocity $\sigma_{U}$, such that for example, the mean sea level (set-up) is

$<r>=\frac{\sigma_{U}^{2}}{2 g}$.

Using assumptions of the Gaussian stationary process for the incident wave, expressions for variance $\sigma_{r}$, skewness $s$ and kurtosis $k$ of the nonlinear wave field at the beach have been established in (Didenkulova et al, 2011):

$\sigma_{r}^{2}=<r^{2}>-<r>^{2}=\sigma_{R}^{2}-2<r>^{2}$.

$s=\frac{<(r-<r>)^{3}>}{\sigma_{r}^{3}}=\frac{8<r>^{3}}{\left(\sigma_{R}^{2}-2<r>^{2}\right)^{3 / 2}}$,

$k=\frac{<(r-<r>)^{4}>}{\sigma_{r}^{4}}-3=\frac{<r>^{2}\left(4 \sigma_{R}^{2}-23<r>^{2}\right)}{\left(\sigma_{R}^{2}-2<r>^{2}\right)^{2}}$.

At the same time, the random functions $R(t)$ and $U(t)$ are connected

$U=\frac{1}{\alpha} \frac{d R}{d t}$

and, therefore, they do not correlate with each other, hence, standard deviations $\sigma_{R}$ and $\sigma_{U}$ should be determined independently. Thus, Eqs. (3)-(6) can be expressed in a nondimensional form with the use of a single parameter $\mathrm{Br}_{\sigma}$ (wave breaking parameter)

$\mathrm{Br}_{\sigma}=\frac{\sigma_{U}^{2}}{g \sigma_{R}}, \quad \frac{<r>}{\sigma_{R}}=\frac{\mathrm{Br}_{\sigma}}{2}, \quad \frac{\sigma_{r}}{\sigma_{R}}=\sqrt{1-\frac{\mathrm{Br}_{\sigma}^{2}}{2}}$,

$s=\frac{\mathrm{Br}_{\sigma}^{3}}{\left[1-\frac{\mathrm{Br}_{\sigma}^{2}}{2}\right]^{3 / 2}}, \quad k=\frac{\mathrm{Br}_{\sigma}^{2}\left[1-\frac{23}{16} \mathrm{Br}_{\sigma}^{2}\right]}{\left[1-\frac{\mathrm{Br}_{\sigma}^{2}}{2}\right]^{2}}$

The parameters $\sigma_{R}$ and $\sigma_{U}$ characterizing the linear wave field in the point $x=0$ are not directly measured in experimental studies, thus, it is more convenient to express Eqs. (8) through measured characteristics $\sigma_{r}$ and $\sigma_{u}$

$\mathrm{Br}_{\sigma}=\frac{\sigma_{u}^{2}}{g \sigma_{r}} \frac{1}{\sqrt{1+\frac{1}{2}\left(\frac{\sigma_{u}^{2}}{g \sigma_{r}}\right)^{2}}}, \quad \frac{<r>}{\sigma_{r}}=\frac{\mathrm{Br}_{\sigma}}{2 \sqrt{1-\mathrm{Br}_{\sigma}^{2} / 2}}$,

and analyze experimental results with respect to the shoreline displacement and velocity.

The velocity of the moving shoreline has a Gaussian distribution if the wave field offshore is also described by the 
Gaussian distribution. However, the distribution of the displacement of the moving shoreline is non-Gaussian. If the deviation is weak (small values of the parameter $\mathrm{Br}_{\sigma}$ ), its probability density function can be found by a perturbation technique based on the Gram-Charlier series of Type A (Kendall and Stuart, 1969; Massel, 1996).

$\xi=\frac{r}{\sigma_{r}}, \quad w_{r}\left(\xi, \mathrm{Br}_{\sigma}\right)=f(r) \sigma_{r}$,

The probability density function $w_{r}$ in this case can be represented as

$$
\begin{aligned}
& w_{r}\left(\xi, \mathrm{Br}_{\sigma}\right)=\sqrt{\frac{1}{2 \pi}} \exp \left[-\frac{\Psi^{2}}{2 \Sigma}\right] \\
& \left\{1+\frac{s\left(\mathrm{Br}_{\sigma}\right)}{3 !} H_{3}\left(\frac{\Psi}{\sqrt{\Sigma}}\right)+\frac{k\left(\mathrm{Br}_{\sigma}\right)}{4 !} H_{4}\left(\frac{\Psi}{\sqrt{\Sigma}}\right)+\ldots\right\},
\end{aligned}
$$

where $H(\rho)$ are the Hermite polynomials

$$
H_{3}(\rho)=\rho^{3}-3 \rho, \quad H_{4}(\rho)=\rho^{4}-6 \rho^{2}+3 .
$$

and

$\Psi=\xi-\frac{\mathrm{Br}_{\sigma}}{2}, \quad \Sigma=1-\frac{\mathrm{Br}_{\sigma}^{2}}{2}$.

The probability density function $w_{r}$ is shown in Fig. 1 for several values of the parameter $\mathrm{Br}_{\sigma}$. It is evident that $w_{r}$ becomes asymmetric and shifts towards large values of shoreline displacement $\xi$ with an increase in parameter $\mathrm{Br}_{\sigma}$.

Figure 1 includes both linear and semi-logarithmic scales of the distribution in order to demonstrate the changes in the main body (slight increase of the mean level) and in the tails (the probability of extreme runups increases and the probability of extreme backwashes decreases). This demonstrates that wave runup prevails over the backwash even in cases when the incident wave is symmetrical with respect to the horizontal axis. This suggests, that nonlinear waves will cause more prolonged flooding at the coast.

\section{Experimental setup}

Experimental investigations were completed in the new wave flume at the University of Warwick, of dimensions, $22 \mathrm{~m}$ long, $0.6 \mathrm{~m}$ wide and an operating water depth of $0.5 \mathrm{~m}$. The channel is equipped with an absorbing-piston type wavemaker (Spinneken and Swan, 2009). The wavemaker paddle is equipped with an active absorption mechanism, such that it is assumed that the runup statistical processes can be treated as stationary. Experiments were conducted on a plain 1:3.4 impermeable beach, located at the far end of the flume (Fig. 2). Water surface elevations were measured by resistance probes installed at 7 locations throughout the flume (location $x=4,4.4,4.8 \ldots 6.4 \mathrm{~m}$ from the slope). Probes were spaced by $0.4 \mathrm{~m}$ to span at least a half-wavelength to reconstruct the incident wave from its superposition with the wave reflected by the beach (the method explained below).
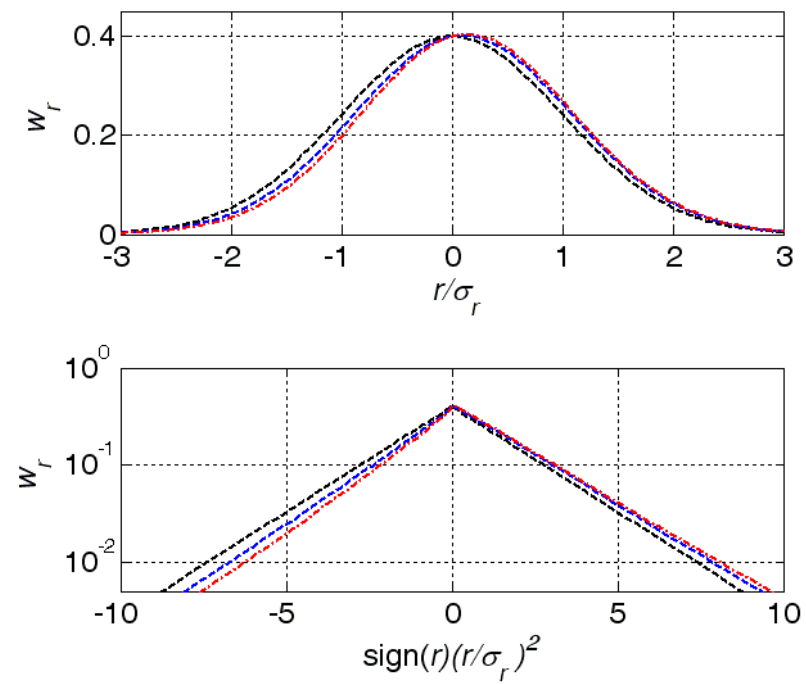

Fig. 1. Probability density function of the displacement of the moving shoreline for $\mathrm{Br}_{\sigma}=0$ (solid line), $\mathrm{Br}_{\sigma}=0.2$ (dashed line) and $\mathrm{Br}_{\sigma}=0.3$ (dash-dotted line).

The runup was measured by a capacitance probe consisting of the two lacquered copper wires of $0.2 \mathrm{~mm}$ thick suspended in tension at $5 \mathrm{~mm}$ above the slope. The fluid velocity at the location of the wires is nearly parallel to the slope, hence the flow doesn't significantly deflect the wires. The distance between wires $(20 \mathrm{~cm})$ is large compared with the possible displacement due to the water motion and combined with the logarithmic decay of electric field provides vanishingly small effect on the probe reading. A 5 volt $100 \mathrm{kHz}$ signal was applied to the one of the wires. The signal from the other wire was treated by a lock-in amplifier and its amplitude was logged with the sampling frequency of $200 \mathrm{~Hz}$. The signal from wave gauges was recorded with the sampling frequency of $128 \mathrm{~Hz}$. To speed up the processing, both signals were decimated to $32 \mathrm{~Hz}$ as 64 sampling points per wave period is commonly considered sufficient. Calibration of the probe was performed by comparing the signal with results of video-recording. To avoid the drift of both runup and the wave probes, water was kept in the channel for 5 days before the experiment, to stabilize its temperature and thus concentration of dissolved gases which can strongly affect conductivity. The shoreline speed was calculated as the time derivative of the runup signal.

The reason for not placing the capacitance probe directly on the slope was the evidence of a thin layer of water (order $1 \mathrm{~mm}$ ), which waves leave on retraction. This would have affected the capacitance between wires placed on the slope. Suspension of the wires by $5 \mathrm{~mm}$ results in an error of the similar magnitude in runup measurement. This is comparable with the error introduced by the capillary effect at the shoreline. To estimate the latter, we set the capillary pressure associated with meniscus formation equal to the hydrostatic pressure, i.e. $\frac{\sigma}{h / 2} \approx \rho g h$ which results in $h \approx \sqrt{\frac{2 \sigma}{\rho g}} \approx 3 \mathrm{~mm}$. 


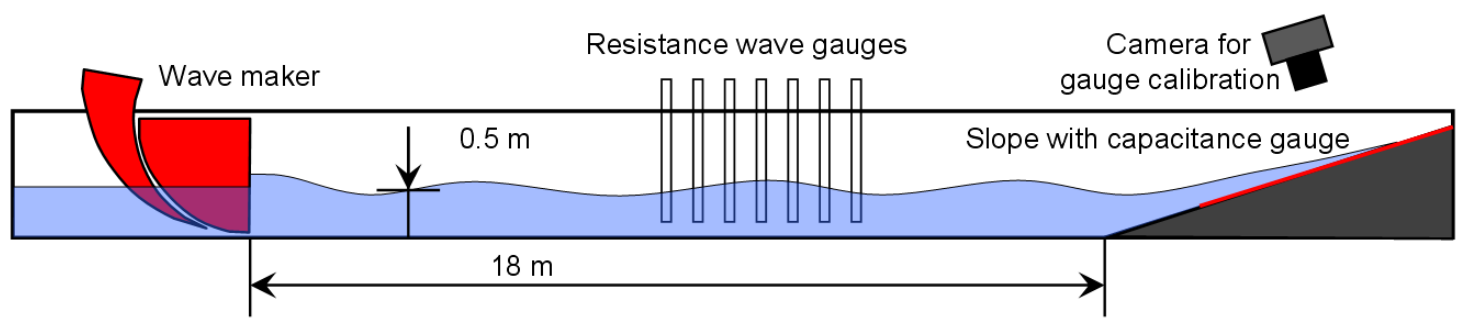

Fig. 2. Experimental setup.
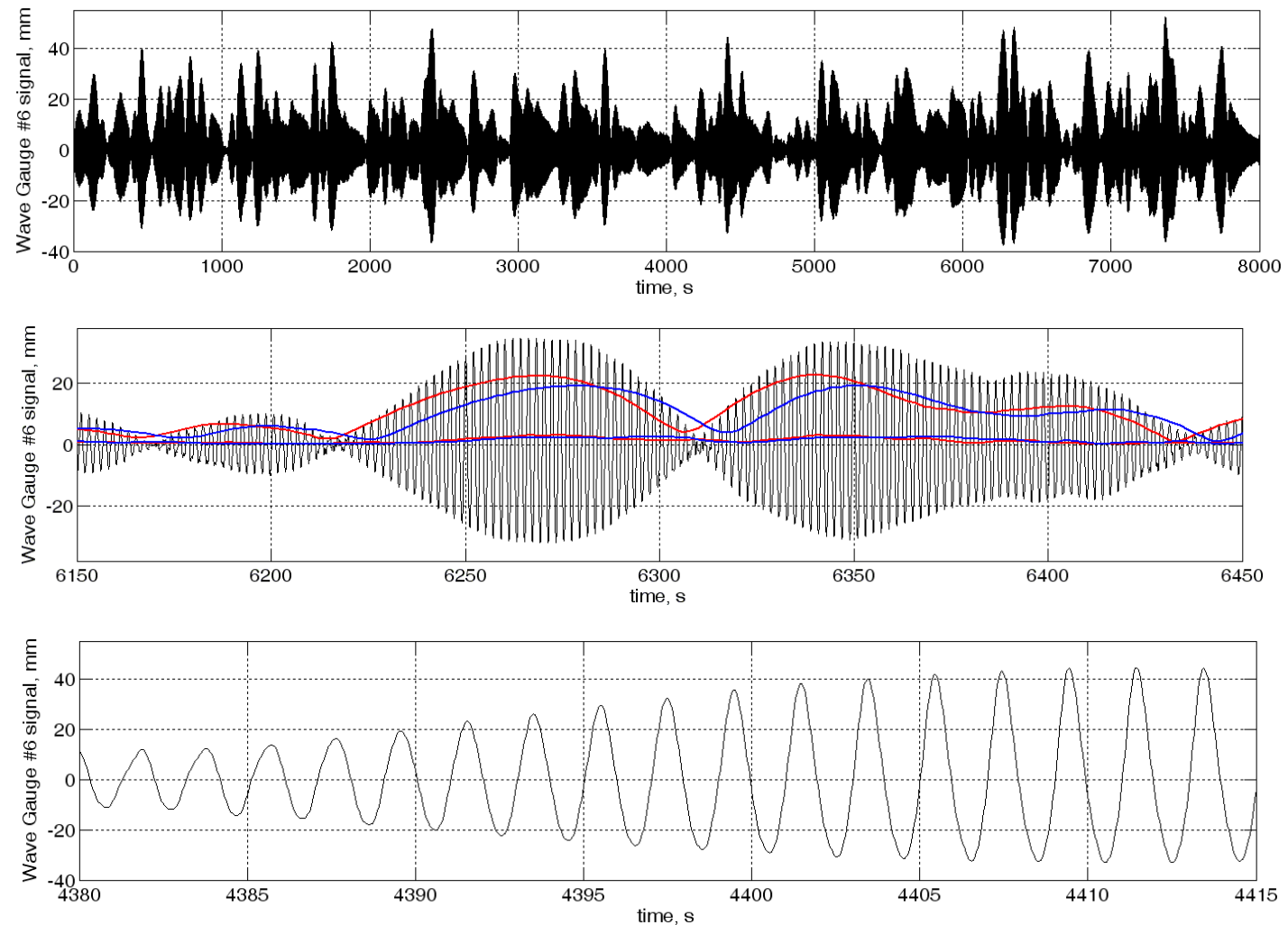

Fig. 3. Top: a typical wave gauge signal; Middle: zoomed signal with reconstructed amplitudes of first and second harmonics of the incident (red) and reflected (blue) waves; Bottom: zoomed signal from the wave gauge.

Another source of error affecting the measurements was formation of a viscous boundary layer at the slope. To evaluate the layer thickness, we use the standard estimate for a viscous boundary layer forming during the wave half-period $h \approx \sqrt{v \frac{1}{2} \frac{2 \pi}{\omega}} \approx 1 \mathrm{~mm}$. As $h \rightarrow \infty$ as $\omega \rightarrow 0$, we note that the viscosity plays its role only when the shoreline speed is greater or comparable with the speed of water flowing down due to gravity in a layer of the thickness $h$, i.e. when $\frac{\omega R}{\sin \alpha} \approx \frac{g \sin \alpha}{2 v} h^{2}$, which is true for the higher end of amplitudes we deal with.

Generation of the narrow band spectrum at $0.5 \mathrm{~Hz}$ was provided by simultaneously generating 32 monochromatic waves of equal amplitudes and the frequencies evenly spaced in the interval $0.488-0.512 \mathrm{~Hz}$. The wave pattern has been generated at several amplitudes for the duration of $8000 \mathrm{~s}$ each. Thus, statistics over approximately 4000 waves was collected.

The experiments were designed for non-breaking conditions, the wave number times depth $k h=0.774$, which is less than 1, thus satisfying the shallow water approximation. Visual observation of waves has been performed to ensure breaking was not occurring. 

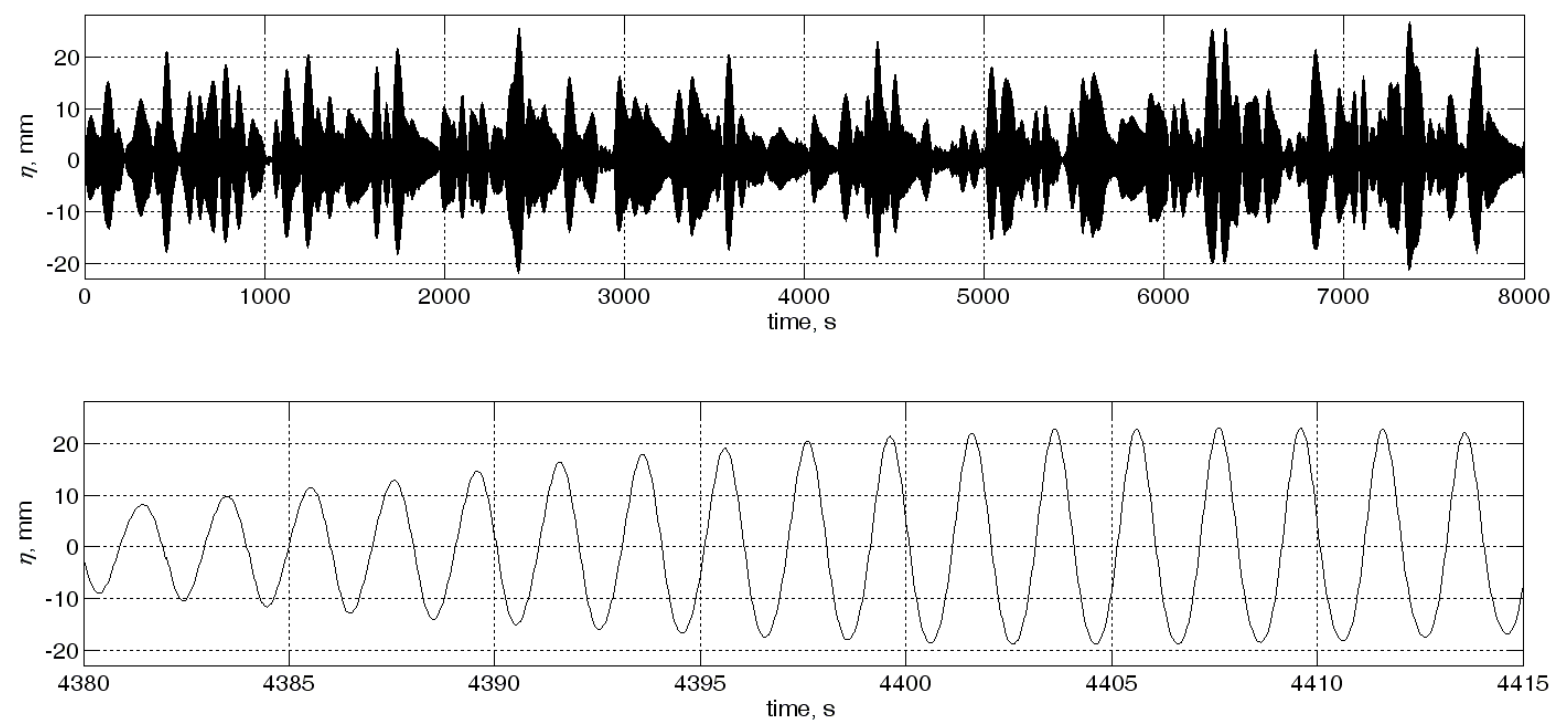

Fig. 4. Reconstructed incident wave (top) and its zoom (bottom).
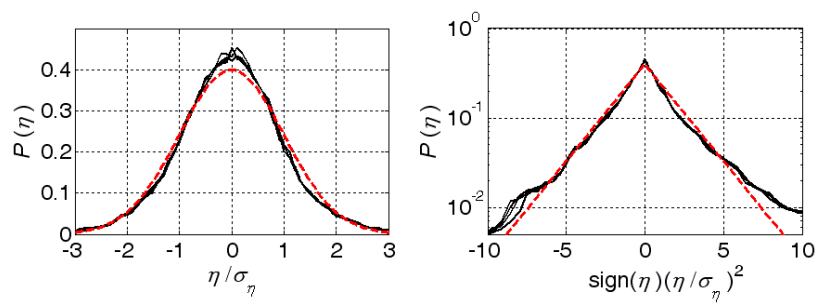

Fig. 5. Probability density function of the incident wave field in linear and semi-logarithmic scales. Solid lines show the statistics for experiments with $\mathrm{Br}_{\sigma}=0.2,0.25,0.27$, and 0.3. Dashed line corresponds to the normal distribution.

\section{Incident wave field}

To compare statistics of the runup height to that of the incident waves, the incident waveform has been extracted from the signals at 7 evenly spaced resistance gauges using the first two Fourier harmonics of the signals. The method used is similar to that described in Goda and Suzuki (1976). Let the gauge coordinates are $x i, i=1 . .6$, frequencies of signal harmonics are $n \omega_{0}$. Then, the water elevation at a gauge $i$ can be written in the form

$$
\begin{aligned}
& \eta_{i}=\sum_{n=1,2}\left[A_{n}^{c} \cos \left(k_{n} x_{i}-n \omega_{0} t\right)+A_{n}^{s} \sin \left(k_{n} x_{i}-n \omega_{0} t\right)+\right. \\
& \left.B_{n}^{c} \cos \left(-k_{n} x_{i}-n \omega_{0} t\right)+B_{n}^{s} \sin \left(-k_{n} x_{i}-n \omega_{0} t\right)\right] \\
& =\sum_{n=1,2}\left[F_{\mathrm{in}}^{c} \cos n \omega_{0} t+F_{\mathrm{in}}^{s} \sin n \omega_{0} t\right]
\end{aligned}
$$

where $A_{n}^{c}, A_{n}^{s}, B_{n}^{c}, B_{n}^{s}$ are coefficients of $n$-th $\cos$ and $\sin$ harmonics of the incident and the reflected waves, and $F_{\text {in }}^{c}$, $F_{\text {in }}^{s}$ are the $n$-th cos and sin coefficients of the time series of water elevation at $n$-th gauge. Rearranging the trigonometric terms and equating coefficients of $\cos \left(n \omega_{0} t\right)$ and $\sin \left(n \omega_{0} t\right)$ at similar $n$, we get a pair of equations for each probe:

$$
\begin{aligned}
& A_{n}^{c} \cos k_{n} x_{i}+A_{n}^{s} \sin k_{n} x_{i}+B_{n}^{c} \cos k_{n} x_{i}-B_{n}^{s} \sin k_{n} x_{i}=F_{\mathrm{in}}^{c} \\
& A_{n}^{c} \sin k_{n} x_{i}-A_{n}^{s} \cos k_{n} x_{i}-B_{n}^{c} \sin k_{n} x_{i}-B_{n}^{s} \cos k_{n} x_{i}=F_{\mathrm{in}}^{s}
\end{aligned}
$$

Hence, by Fourier-decomposing the signal from 2 probes, for each harmonic $n$ we get a system of 4 equations involving 4 variables $A_{n}^{c}, A_{n}^{s}, B_{n}^{c}, B_{n}^{s}$, and are able to recover every harmonics of the incident wave and thus to reconstruct the incident signal. In reality, precision of the measurement allows only to recover first two harmonics which gives a good approximation for the incident wave. To utilize the existence of the 7 probes, we have averaged results obtained from the pairs for which the determinant of the system composed of the equations like Eq. (17) is greater than 2. As the generated wave was of a narrow band spectrum, by applying the Fourier transform to 1-period (2s) window of a signal, we were able to reconstruct the incident wave of varying amplitude and analyze its statistics.

Experimental runs corresponding to the incident wave root mean square $\mathrm{RMS}=2.0,2.5,2.7$, and $3.1 \mathrm{~mm}$ have been analysed. A typical signal from the wave gauge is shown in Fig. 3. The amplitudes of first and second harmonics of incident and reflected waves are imposed on the gauge signal. The front-back asymmetry of weak amplitude waves can be seen in the beginning of the zoomed part of the record and the non-linear peaks are seen where the waves become large, which demonstrates existence of higher harmonics. In average, the 2 nd harmonic constitutes approximately $8 \%$ of the total amplitude of the incident wave and this value is nearly constant through all runs. The delay between the peak of reflected wave amplitude and that of the incident wave 

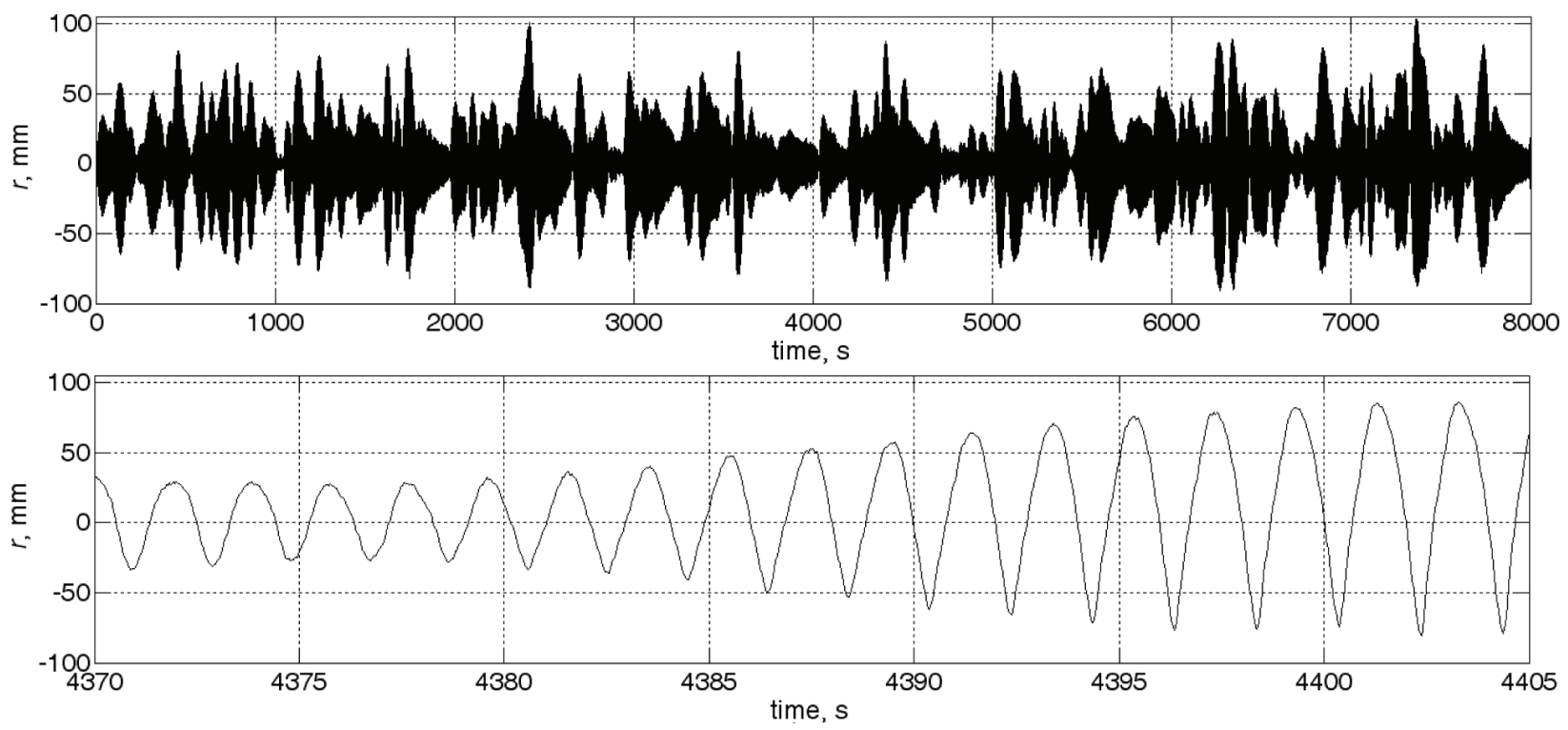

Fig. 6. Typical signals from the runup probe (top) and its zoom (bottom).
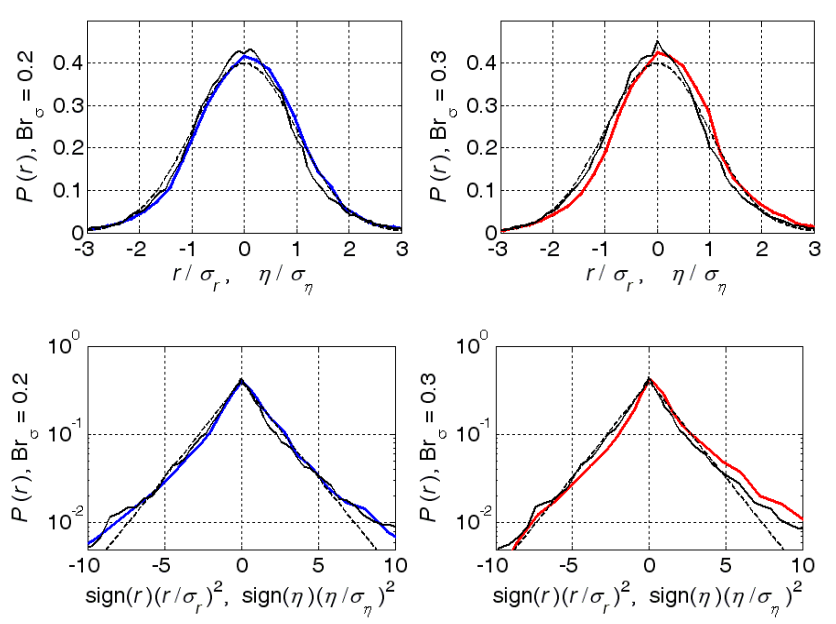

Fig. 7. Probability density functions of wave runup and the incident wave elevation in linear (top) and semi-logarithmic (bottom) scale. Colour lines correspond to runup statistics and black lines show distribution of the water elevation in the incident wave which is separately plotted in Fig. 5. Black dashed lines show the Normal distribution.

corresponds to the distance between the probe array and the slope. Delay between the peak of the second harmonic and that of the first harmonic corresponds to the lower speed of shorter waves generated by the wavemaker because the amplitude of its panel motion is independent of the vertical coordinate. In Fig. 4, the reconstructed incident wave is shown. Expectedly, the reconstructed wave has smaller amplitude than the wave signal (Fig. 3) and repeats its behavior.
The probability density function (pdf) of the incident wave field is shown in Fig. 5. It can be seen that the distribution slightly deviates from Gaussian, which is also confirmed by non-zero values of skewness (about 0.1) and kurtosis (up to 2.5). For clarity, the quadratic semi-logarithmic scale has been applied to the horizontal axis.

\section{Wave runup}

A typical runup signal is shown in Fig. 6. The general structure of the signal repeats the one from the incident wave (Fig. 4). Here, the maximum runup amplitude exceeds the maximum amplitude of the incident wave 4 times. It also can be seen that the waves on the beach become more nonlinear with increases in their amplitude and it is manifested in a parabolic shape of the water displacement at the runup stage and a sharp beak shape during the backwash. Runup of weak-amplitude waves is more sinusoidal.

The corresponding pdf of wave runup is shown in Fig. 7 for two different values of the parameter $\mathrm{Br}_{\sigma}$, which were defined by Eq. (10) from the measured runup field. The velocity of the moving shoreline was found as a time derivative of vertical runup displacement (the same as Eq. 7). A shift of the distribution towards the positive runup heights is evident and agrees with the theoretical results obtained for the narrow-band Gaussian field. For $\mathrm{Br}_{\sigma}=0.2$ the runup distribution almost repeats the distribution for the incident wave, while with increase in $\mathrm{Br}_{\sigma}\left(\mathrm{Br}_{\sigma}=0.3\right)$ the distribution becomes more asymmetric and its maximum increases and shifts more to positive values, which can be seen from the linear figure (top of Fig. 7). At the same time the probability of larger runups increases with increase in $\mathrm{Br}_{\sigma}$, this effect 

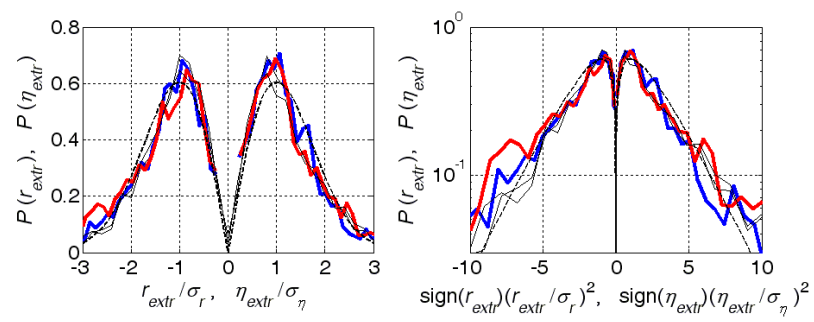

Fig. 8. Statistics of runup amplitudes. Colour lines correspond to runup distributions with $\mathrm{Br}_{\sigma}=0.2$ (blue), and 0.3 (red). Statistics of the incident wave field is indicated by black lines. Dashed line corresponds to Rayleigh distribution.

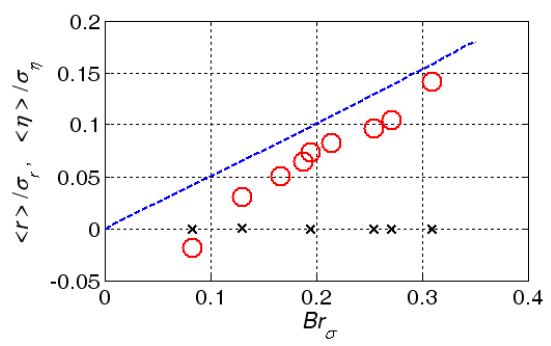

Fig. 9. Mean sea level for different values of $\mathrm{Br}_{\sigma}$. Circles correspond to runup, crosses to the incident wave, and dashed lines to the theoretical Eq. (10).

is more pronounced in the semi-logarithmic scale (bottom of Fig. 7). All this is in a good agreement with an asymptotic description Eq. (12), presented in Fig. 1.

In Fig. 8, we present probability density functions of runup and incident wave heights, which are normalized appropriately and plotted on the same axis. The horizontal axis of incident wave distribution is normalized by its standard deviation $\sigma_{\eta}$, horizontal axis of the runup (negative part for the backwash) distribution is normalized by the corresponding standard deviation $\sigma_{r}$.

Although statistics of extreme values of the incident wave is not described by the Rayleigh distribution and neither is the statistics of the runup height (Fig. 8), both distributions appear almost identical, which confirms the theoretical result that nonlinear wave propagation in the coastal zone does not change statistics of extremes.

It can be seen in Fig. 8 that the most pronounced difference between incident wave amplitudes and the extreme runup values occur at the deepest backwash stage, where the first wave breaking naturally occurs (Zahibo et al., 2008). It is worth noting that experiments were conducted in regimes short of wave breaking where theoretical assumptions may cease to be valid.

The mean water level of the incident wave field and runup are shown in Fig. 9, the dashed lines correspond to theoretical Eq. (10). The incident wave field was generated with a zero
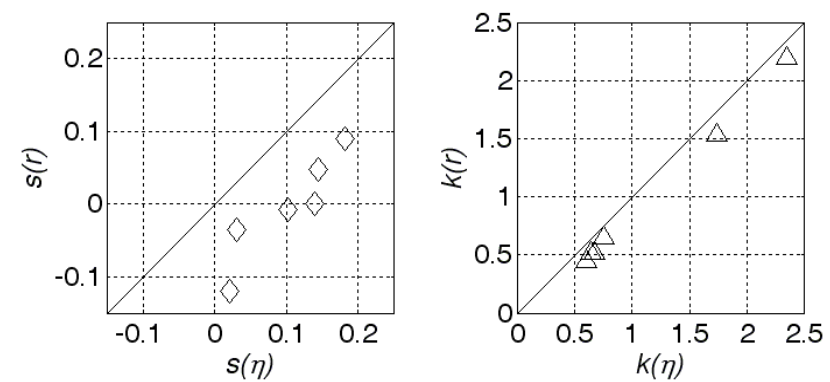

Fig. 10. Skewness and kurtosis of wave runup distribution with respect to those for the incident wave field.
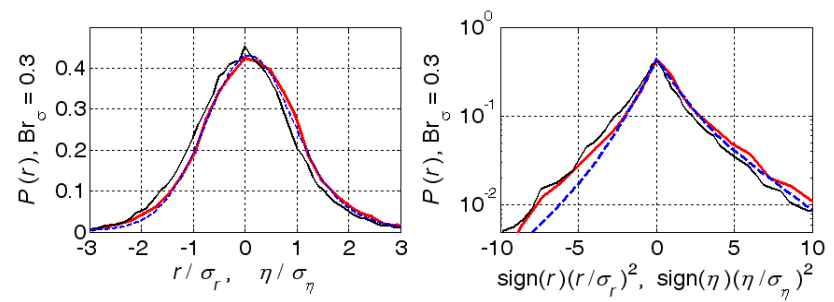

Fig. 11. Red lines correspond to runup statistics and black lines show distribution of the water elevation in the incident wave for $\mathrm{Br}_{\sigma}=0.3$. The dashed blue lines represent the forecasted probability density functions of wave runup calculated from Eq. (12) by substituting measured values for skewness and kurtosis instead of those predicted by Eq. (9).

mean, while the mean water level on the beach grows with an increase in $\mathrm{Br}_{\sigma}$, which agrees with the theory.

Figure 10 shows the relationship between higher statistical moments (skewness and kurtosis) for incident wave field and waves on the beach. It can be seen that values of both skewness and kurtosis for wave runup, only slightly deviate from those for the incident wave field. Therefore, the skewness and kurtosis of the incident wave field could be used to determine the prognostic wave runup distribution by Eq. (12), thus allowing forecasting of runup on actual coastlines.

An example of such forecast is shown in Fig. 11 for for $\mathrm{Br}_{\sigma}=0.3$, where the forecasted probability density functions of wave runup calculated from Eq. (12) by substituting measured values for skewness and kurtosis instead of those predicted by Eq. (9) is compared with the measured statistics of the incident wave and wave runup. It can be seen that the forecast gives a good fit for weak and moderate amplitude waves and demonstrate some reasonable deviations for waves of extreme amplitudes. The largest discrepancy between measured and forecasted data is observed at the backwash stage. This can be related to the wave breaking effects, which occur at the backwash stage for shallow water waves (Zahibo et al., 2008).

The probability density function of the shoreline velocity $w_{u}$ is shown in Fig. 12. The dashed line corresponds to the normal distribution. Although the function $w_{u}$ 

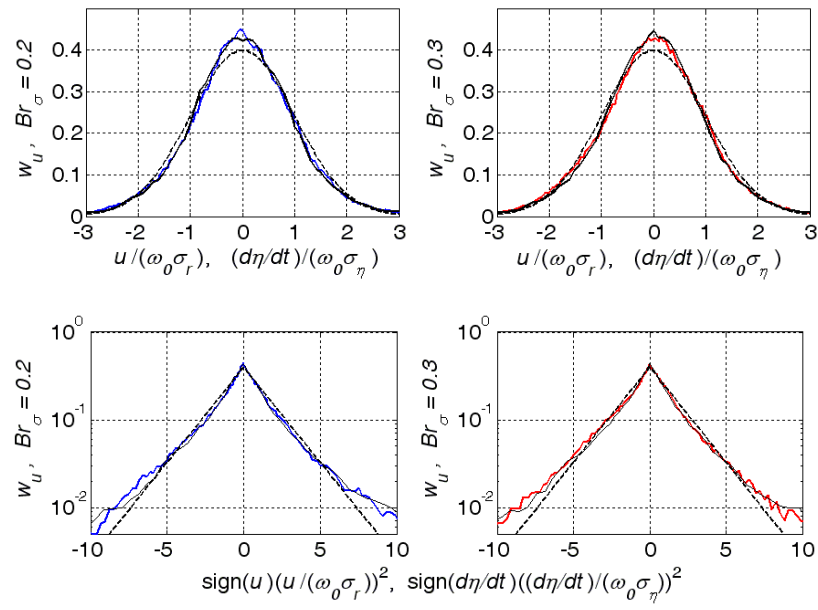

Fig. 12. Probability density function of the shoreline speed in linear (top) and semi-logarithmic (bottom) scale. Colour lines correspond to statistics of the moving shoreline and black lines show distribution of the vertical velocity in the incident wave. Black dashed lines show Normal distribution.

slightly deviates from the normal distribution and asymmetry between positive and negative velocities is clearly seen in the semi-logarithmic plot, the statistics of the shoreline velocity is in a good agreement with the statistics of the vertical velocity in the incident wave.

\section{Conclusions}

This paper represents a study on how the nonlinearity, which is associated with the wave amplitude and a breaking parameter, influences the statistics of long waves at the coast. This is a typical situation for many natural coasts affected by swell, storms and sometimes even tsunamis after a long time of their propagation, and has many practical applications. This paper presents the set-up, skewness and kurtosis as a function of observed non-linear runup characteristics, which is convenient for experimental investigations and differs from those connections introduced in (Didenkulova et al., 2011). However, the importance of this work is in an experimental study of long irregular waves in laboratory conditions, which is usually considered only for short breaking waves, while for long waves only deterministic waves are studied.

The runup of long irregular waves on a plane beach is studied experimentally in the wave flume at the University of Warwick. The case of narrow band spectrum has been studied. Displacement and velocity of the moving shoreline and their amplitudes are analyzed with respect to the amplitude of the incident wave field (different values of the wave breaking parameter $\mathrm{Br}_{\sigma}$ ). It is shown that statistics of the shoreline velocity coincides with the statistics of velocity in the incident wave field, which agrees with the theory (Didenkulova et al., 2011). Distribution of runup amplitudes is also similar to that of the incident wave amplitudes.

The experimental research goes beyond theoretical limits and shows tendencies in a wider range. It is confirmed experimentally that the mean sea level at the coast (wave setup) increases with an increase in wave amplitude (parameter $\mathrm{Br}_{\sigma}$ ) as predicted by Didenkulova et al. (2011). The higher statistical moments (skewness and kurtosis) of water elevation at the coast depend on the parameters of the incident wave field and are hard to forecast with a theoretical assumption of narrow-band Gaussian process. However, their values change consistently with those of incident wave field, and might be used for building prognostic distributions of the beach flooding.

Acknowledgements. Partial support from the targeted financing by the Estonian Ministry of Education and Research (grant SF0140007s11), Estonian Science Foundation (grant 8870), RFBR grants (11-05-00216, 11-02-00483, 11-05-92002, 11-05-97006) and grants MK-1440.2012.5 and MK-4378.2011.5 is greatly acknowledged.

Edited by: A. Slunyaev

Reviewed by: two anonymous referees

\section{References}

Bowen, A. J., Inman, D. L., and Simmons, V. P.: Wave "set-down" and "set-up", J. Geophys. Res., 73, 2569-2577, 1968.

Carrier, G. F. and Greenspan, H. P.: Water waves of finite amplitude on a sloping beach, J. Fluid Mech., 4, 97-109, 1958.

Choi, B. H., Kim, D. C., Pelinovsky, E. and Woo, S. B.: Threedimensional simulation of tsunami run-up around conical island, Coast. Eng., 54, 618-629, 2007.

Choi, B. H., Pelinovsky, E., Kim, D. C., Didenkulova, I., and Woo, S.-B.: Two- and three-dimensional computation of solitary wave runup on non-plane beach, Nonlin. Processes Geophys., 15, 489502, doi:10.5194/npg-15-489-2008, 2008.

Dean, R. G. and Walton, T. L.: Wave setup, in: Handbook of coastal and ocean engineering, edited by: Kim, Y. C., World Sci., Singapore, 2009.

Didenkulova, I., Pelinovsky, E., and Sergeeva, A.: Statistical characteristics of long waves nearshore, Coast. Eng., 58, 94-102, 2011.

Didenkulova, I., Pelinovsky, E., and Soomere, T.: Run-up characteristics of tsunami waves of "unknown" shapes, Pure Appl. Geophys., 165, 2249-2264, 2008.

Didenkulova, I., Pelinovsky, E., Soomere, T., and Zahibo, N.: Runup of nonlinear asymmetric waves on a plane beach, Tsunami and Nonlinear Waves, edited by: Anjan Kundu, Springer, 173-188, 2007.

Didenkulova, I. I., Zahibo, N., Kurkin, A. A., Levin, B. V., Pelinovsky, E. N., and Soomere, T.: Runup of nonlinearly deformed waves on a coast, Doklady Earth Sci., 411, 1241-1243, 2006 b.

Fuhrman, D. R. and Madsen, P. A.: Simulation of nonlinear wave run-up with a high-order Boussinesq model, Coast. Eng., 55, 139-154, 2008. 
Goda, Y. and Suzuki, Y.: Estimation of Incident and Reflected Waves in Random Wave Experiments. Proceedings, 15th Internation Conference on Coastal Engineering, Honolulu, Hawaii., $1,828-845,1976$.

Guza, R. T. and Thornton, E. B.: Local and shoaled comparisons of sea surface elevations, pressures, and velocities, J. Geophys. Res., 85, 1524-1530, 1980.

Holman, R. A.: Extreme value statistics for wave run-up on a natural beach, Coast. Eng., 9, 527-544, 1986.

Holman, R. A. and Sallenger, A. H.: Setup and Swash on a Natural Beach, J. Geophys. Res., 90, 945-953, 1985.

Huntley, D. A., Guza, R. T., and Bowen, A. J.: A universal form for shoreline run-up spectra, J. Geophys. Res., 82, 2577-2581, 1977.

Kendall, M. G. and Stuart, A.: The advanced theory of statistics, Volume I., Distribution theory, London, 439 pp., 1969.

Kennedy, A. B., Chen, Q., Kirby, J. T., and Dalrymple, R. A.: Boussinesq modeling of wave transformation, breaking and runup, J. Waterway, Port Coast. Ocean Eng. 126, 39-47, 2000.

Le Mehaute, B., Koh, R. C., and Hwang, L. S.: A synthesis of wave run-up, J. Waterways Port Harbor Coast. Eng., 94, 77-92, 1968.

Liu, P. L.-F., Cho, Y.-S., Briggs, M. J., Kanoglu, U., and Synolakis, C.: Runup of solitary waves on a circular island, J. Fluid Mech., 302, 259-285, 1995.
Mansard, E. P. D. and Funke E. R.: The measurement of incident and reflected spectra using a least square method, in: Proc. 17th Coastal Eng. Conf., ASCE, 154-172, 1980,

Massel, S. R.: Hydrodynamics of coastal zones, Elsevier, Amsterdam, 1989.

Massel, S. R.: Ocean surface waves: their physics and prediction, World Scientific Publ., Singapore, 492 pp., 1996.

Raubenneimer, B. and Guza, R. T.: Observations and predictions of run-up, J. Geophys. Res., 101, 25575-25587, 1996.

Raubenneimer, B., Guza, R. T., and Elgar, S.: Field observations of wave-driven setdown and setup, J. Geophys. Res., 106, 46394638, 2001.

Stockdon, H. F., Holman, R. A., Howd, P. A., and Sallenger, A. H.: Empirical parameterization of setup, swash, and runup, Coast. Eng., 53, 573-588, 2006.

Spinneken, J. and Swan, C.: Second-order wave maker theory using force-feedback control, Part I. A new theory for regular wave generation, Ocean Eng., 36, 539-548, 2009.

Synolakis, C .E.: Tsunami runup on steep slopes: How good linear theory really is, Nat. Hazards, 4, 221-234, 1991.

Zahibo, N., Didenkulova, I., Kurkin, A., and Pelinovsky, E.: Steepness and spectrum of nonlinear deformed shallow water wave, Ocean Eng., 35, 47-52, 2008. 